\title{
Hydro-mechanical behavior of fractures: 2D, F.E.M. modelling
}

\author{
C. Guiducci \\ Scuola Normale Superiore di Pisa - Italia (Italy)
}

\author{
A. Pellegrino \\ Eni - Agip Division - Italia (Italy) \\ J.P. Radu, F. Collin, R. Charlier \\ Département GéomaC - Université de Liège - Belgique (Belgium)
}

\begin{abstract}
A numerical approach for modeling the coupled hydro-mechanical fracture behavior is proposed. The movement of fluids through rock fractures and consequently the evolution of their hydraulic conductivity is an important subject in many petroleum related activities, mainly concerning the change of well productivity during the reservoir life. It is well known that the flow in the fractures is strongly controlled by the fracture apertures. Recent investigations on the distribution of the apertures in natural fractures suggest that the cubic law can, better than the Darcy law, predict the fluid flux through rough walled fractures as long as the appropriate average fracture aperture is used. A finite element code is developed to predict the influence that the stresses variation in the fracture has on the distributed hydraulic conductivity field. The proposed model combines the stochastic cubic law with a non-linear deformation function (hyperbolic) that is suggested to describe the stress-closure/opening curves of the joints and that allows to couple together the hydraulic and the mechanic fracture behavior. The relationships used and the validity of the present model are tested by means of the comparison between experimental data and numerical predictions (Bart 2000) in various boundary and loading conditions. Comparisons between the proposed new model and a no coupled one have also been performed. They show that the proposed coupled model allows for a more realistic description of the fracture behavior.
\end{abstract}

\section{INTRODUCTION}

A constitutive model is presented in the following paper to simulate the coupled hydro-mechanical behavior of fractures. In particular, starting from a representation of fractures, a good description of hydromechanical behavior of fractured oil reservoir is achieved during the injection/production rate in petroleum engineering applications.

As it's known from the literature, the description of the hydraulic and mechanical behavior of a single fracture is a subject of central importance.

During the last years the main features of the behavior of rock fractures have been studied by the analysis the experimental investigations of many authors like Barton (1976), Bandis \& al (1981), Gentier (1998). A large amount of fracture modeling work is available in the literature. Goodman \& al (1968; 1972), Plesha (1995), Barton \& al (1985) and Bart (2000) are some of the numerous investigators who have derived the basic physical equations describing the fracture behavior. Their numerical investigations have been the basis of our research, which have led to the conception of the proposed model.

So, the numerical model presented reproduces a non-linear coupled fracture behavior when normal effective stresses are applied. The coupling is real- ized combining the cubic law with a non linear deformation function (hyperbolic) to describe the stress-closure/opening curves of the fractures. The coupling behavior under tangential effective stresses is taken into account through the simple Mohr/Coulomb linear relation.

The model was introduced for the interface contact element of the finite element code LAGAMINE implemented by R. Charlier and its group at the University of Liège.

The present paper is mainly divided into fourth parts dealing with:

1) the theoretical aspects of the model, mechanical and hydraulic laws will be presented;

2) the numerical aspects of the finite element LAGAMINE code with particularly attention to the contact element model;

3) an academic application describing a simple geometry of an oil reservoir in order to underline the important hydraulic role of the fractures in a reservoir context;

4) the comparisons between a coupled and a not coupled fracture model in order to show the importance of a coupled representation of the fracture behavior;

5) the sensitivity study showing the power of the model and of the code;

6) conclusions. 


\section{INTERFACE FINITE ELEMENT}

\subsection{General concept of a contact problem}

Consider two deformable solids (or domains) $\Omega^{U}$ and $\Omega^{D}$ with boundaries $\partial \Omega^{U}$ and $\partial \Omega^{D}$ (Fig. 1). They are contacting through boundaries $\partial \Omega_{C}^{U}$ and $\partial \Omega_{C}^{D}$.

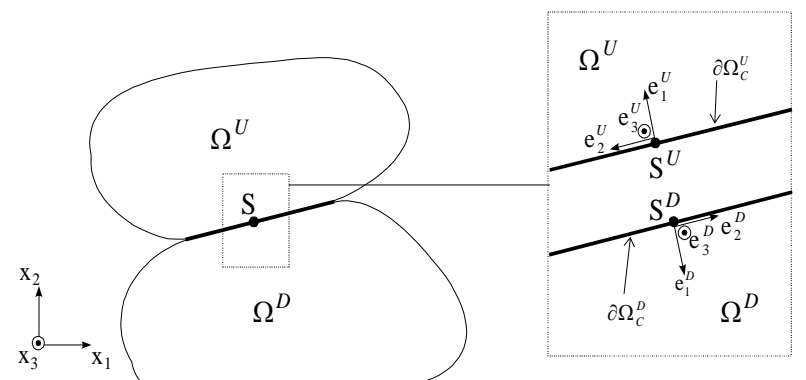

Fig. 1. Contact between 2 deformable solids.

In the local referential plane $\left(e_{1}, e_{2}, e_{3}\right)$ (Fig. 1), for a plane or axi-symmetrical problem, the stress tensor in each solid reduces to a contact stress vector $\underline{\sigma}_{C}$ defined by two components :

$$
\underline{\sigma}_{C}=\left[\begin{array}{l}
\sigma_{1} \\
\sigma_{2}
\end{array}\right]=\left[\begin{array}{c}
-p \\
\tau
\end{array}\right]
$$

where $p$ and $\tau$ are the pressure and the shear objective stress vector (Charlier \& Cescotto 1988). The perfectly sticking contact condition is enforced numerically using the classical penalty method which allows a small relative velocity between points $S^{U}$ and $S^{D}$.

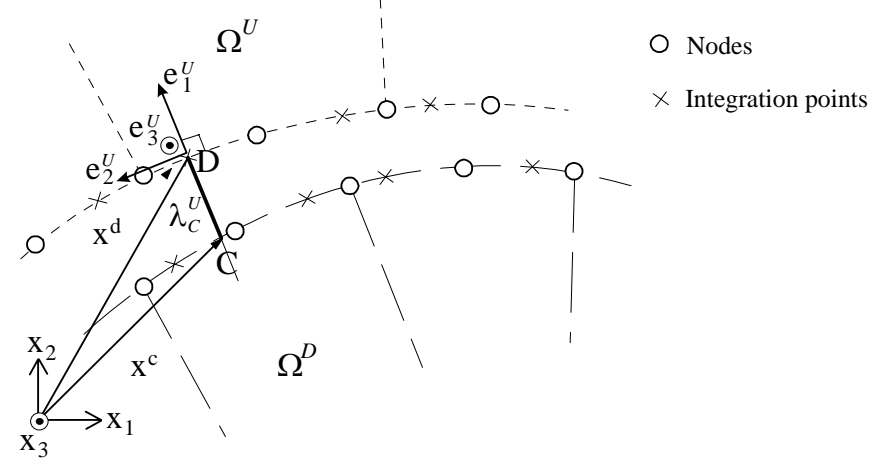

Fig. 2. Parabolic interface finite elements $\left(\lambda^{U}<0\right.$, i.e. no contact).

The contact stress vector $\underline{\sigma}_{C}$ is associated with the relative displacement velocity $\dot{\varepsilon}_{C}$ (through the interface mechanical constitutive law) defined as the time derivative of the distance vector $\mathrm{u}$ between $\partial \Omega_{C}^{U}$ and $\partial \Omega_{C}^{D}$ (Fig. 2).

The contact side of each body $\Omega^{U}$ and $\Omega^{D}$ can be discretised with interface isoparametric elements which are compatible (same degree and common nodes) with the solid finite elements used to discretise the corresponding body (Fig. 2).

The frictional interface elements used here are based on mixed variational (Cescotto \& Charlier 1993): contact stresses are computed at contact ele- ment integration points whereas displacements of the solid boundary are computed at nodal points

In the LAGAMINE code, the reference side $\partial \Omega_{C}$ on which contact stresses are computed is always referred as the contact element side and is discretised using interface elements; the other side on which intersections are looked for is always referred as the foundation side and is discretised using foundation elements.

The contact condition is simply obtained locally from geometrical computation of the distance $\lambda_{C}$ between the two contact interfaces $\partial \Omega_{C}^{U}$ and $\partial \Omega_{C}^{D}$ with $\lambda_{C}=\underline{u} \cdot \underline{e}_{1}$

$-\lambda_{C}<0 \rightarrow$ no contact (see Fig. 3),

- $\lambda_{C} \geq 0 \rightarrow$ there is contact.

For more details see (Habraken \& Cescotto 1996).

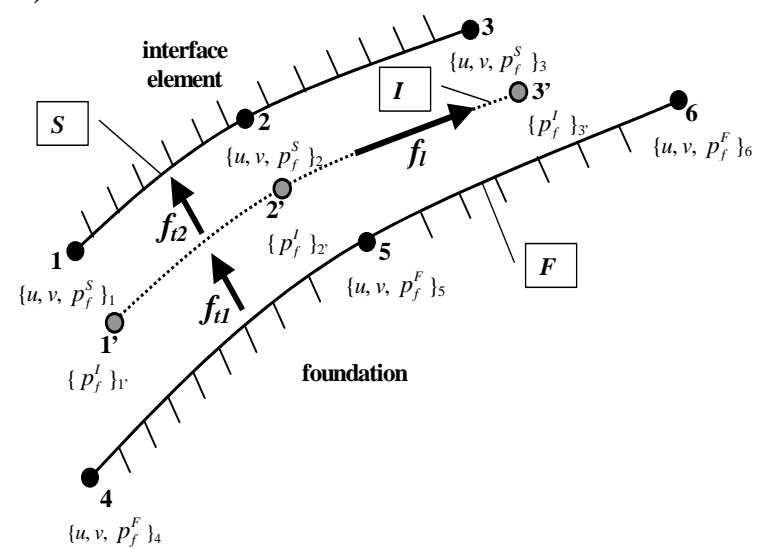

Fig. 3. Description of a 2-D parabolic interface element.

\subsection{Particular description of the interface element}

A 2-D large strain finite element has been implemented in the LAGAMINE code. They are two isoparametric elements (Fig. 3), with 2 (linear) or 3 (parabolic) nodes describing the interface element the foundation side and, with 3 degrees of freedom (d.o.f) per node (2 mechanical displacements $u$ and $\mathrm{v}$, and the fluid pressure on the structure side $p_{f}^{S}$ ). To describe the seepage flow inside the interface $p_{f}^{I}, 2$ or 3 further nodes are added with only 1 d.o.f. per node, the fluid pressure inside the interface; these nodes are thus the same co-ordinates that the corresponding nodes on the interface element.

With that element formulation, the equivalent nodal forces and the stiffness matrix in the NewtonRaphson sense will have, for a parabolic element, the following expression respectively given in (16) and (17) :

$$
\begin{aligned}
& \underline{F}^{T}=\left(\begin{array}{lll}
\left\langle\underline{F}_{S}\right\rangle_{1 \times 9} & \left\langle\underline{F}_{I}\right\rangle_{1 \times 3} & \left\langle\underline{F}_{F}\right\rangle_{1 \times 9}
\end{array}\right)^{T}
\end{aligned}
$$

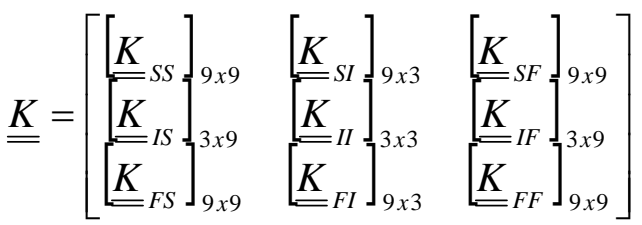


where the indexes $S_{S}{ }_{I}$ and ${ }_{F}$ respectively refer to the solid side, the interior interface and the foundation side.

\subsection{Interface laws}

\subsubsection{Mechanical law}

A particular constitutive law was introduced in the finite element LAGAMINE code to describe the links between the contact stress rate and the contact strain rate of the interface element subjected to normal loading.

This relation, firstly deduced by Goodman's experiments, showed that the fracture closure $\Delta V_{j}$ changes, under increasing normal stress $\left(\sigma_{n}\right)$, in a non-linear way, closing resembling a hyperbola. A characteristic example is illustrated in Fig. 4. The non-linearity in the $\Delta \sigma_{n}-\Delta V_{j}$ relation was also recognized by other authors.

From a physical point of view this behavior can be explained with the progressive mobilization of the fracture asperities. At the beginning of the test, few points are in contact and the deformations related to small imposed stress are important. With the progressive fracture closure changing in the phenomena show that with the increasing augmentation of contact between asperities the relative displacements become smaller with progressing stress applied, until an asymptotic fracture closure value is reached for very high values of stress.

The present behavior can be attributed to the influence of different factors:

a) initial actual contact area and vertical distribution of the aperture between fracture walls;

b) strength and deformability of asperities;

c) fracture wall roughness (Capasso 2000);

d) thickness and physical properties of the unfilling material, if present.

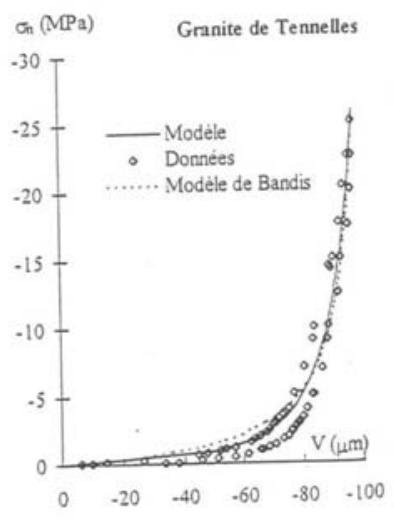

Fig. 4. Normal stress-deformation relations of intact and fractured rock

In this paper the fracture non-linearity in the $\Delta \sigma_{n^{-}}$ $\Delta V_{j}$ relation doesn't take into account the effect of fracture wall aperture, strength, roughness and unfilled materials. Then, the strength-deformation rela- tion is expressed through the empirical hyperbolic function proposed by Bart (2000) (Fig. 4.):

$$
\Delta \sigma_{n}{ }^{\prime}=\frac{K_{n i} D_{0}}{\left(1+\frac{V_{j}}{D_{0}}\right)^{\gamma}} \Delta V_{j}
$$

where:

- $K_{n i}$ is the normal initial stiffness associated to the fracture. This parameter can be obtained as the initial slope of the hyperbola of Fig.4., it's value can be estimated for example starting from the rock matrix damaged stiffness.

- $\quad D_{0}$ is the asympthotical fracture opening, related to the fracture when stresses equal to zero are applied.

- $\quad \gamma$ is an empirical coefficient variable between 2 and 6 , it's value is increasing with the fracture roughness. For that parameter Bandis et al. (1983) proposed the value 2 that seems giving a correct description of the mechanical behavior of the fracture.

\subsubsection{Flow law}

Next step is to model the hydraulic behavior of a loaded fracture. In particular, in LAGAMINE code an anysotropic description of water flows through the interface element is presented. More in details, the fluid flow is described in its transversal and longitudinal path referred to the fracture.

According to the definition of a transverse transmissivity $T_{t}$; two transverse fluid flows $f_{t 1}$ and $f_{t 2}$ can be described from the following relations (Fig. 3):

$$
f_{t 1}=T_{t}\left(p_{f}^{F}-p_{f}^{I}\right) \text { and } f_{t 2}=T_{t}\left(p_{f}^{I}-p_{f}^{S}\right)
$$

describing respectively with $f_{t 1}$ the fluid path from the foundation $F$ to the new internal element $I$ describing the interface and with $f_{t 2}$ the fluid path from the internal element to the proper interface element $S$ (flow moving from one boundary to the other transversally to the fracture).

At the same time, if the interface longitudinal permeability $k_{l}$ is not nil, the longitudinal fluid mass flow $f_{l}$ must be considered. In particular, longitudinal flow through fractures can be assumed analogous to laminar flow between two perfectly smooth parallel plate separated by an uniform distance. This assumption was based on the observation that most natural fractures are approximately planar on the scale of the fracture length. So for laminar flow between two parallel plates, the longitudinal fluid flow referred to the fracture is given by the so called " $\mathrm{cu}$ bic equation” (Tsang and Witherspoon,1981): 
$f_{l}=-d^{3} \frac{\rho_{f} g}{12 \mu_{f}}\left(\underline{\nabla} h_{f}\right)$

where $f_{l}$ varies as the cube of the distance between the plates $d ; \mu_{f}$ is the fluid viscosity, $\rho_{f}$. the constant fluid density and $\Delta \mathrm{h}$ is the change in hydraulic head across the boundaries of the flow domain. As it can be seen in this case the hydraulic conductivity of a fracture with aperture $d$ is given by:

$k_{l}=d^{2} \rho_{f} g / 12 \mu_{f}$

In this case, to describe the fluid flow distribution into the fracture, equations (6) and (7) are introduced into the Reynolds equation, this leads to the following expression:

$\operatorname{div}\left(f_{i}\right)=0$

\section{APPLICATION}

\subsection{Boundary and initial conditions}

Using the presented interface element some applications were developed. In particular, a fluid depletion of a reservoir interested by a horizontal fracture is modeled. The well is situated on the left boundary of the model. For simplicity the fluid in the reservoir is considered to be water and the rock matrix is chalk. The reservoir is modeled in plane strain conditions the dimension being $2500 \mathrm{~m}$ of length by $300 \mathrm{~m}$ of height. The initial fracture opening value is $\approx 0.2 \mathrm{~mm}$ (Fig. 5).

The initial stress field is obtained, neglecting gravity effects, applying $62 \mathrm{MPa}$ overburden load and a $62 \mathrm{MPa}$ horizontal stress imposed on the well boundary. Initial fluid pressure of the reservoir is 48.7 MPa (Fig. 6).

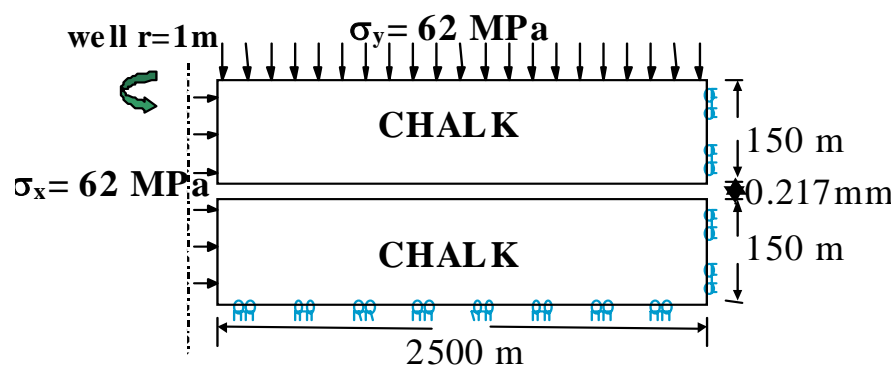

Fig. 5. Mechanical boundary conditions.

A production phase was modeled starting from those initial conditions. A first step of $15 \mathrm{MPa}$ fluid pressure decrease is applied for 7.5 years at the well boundary. A following second step lasting 12.5 years is perceived keeping fluid pressure constant (Fig. 7).

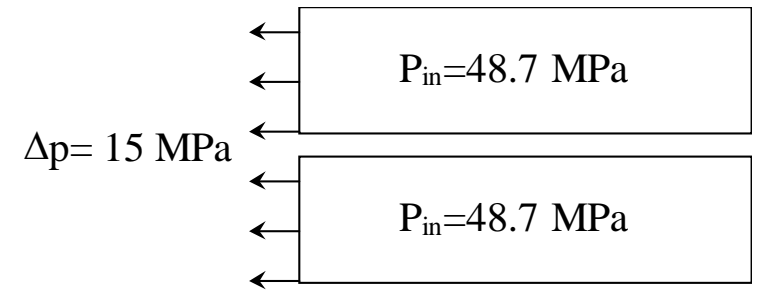

Fig. 6. Hydraulic boundary conditions.

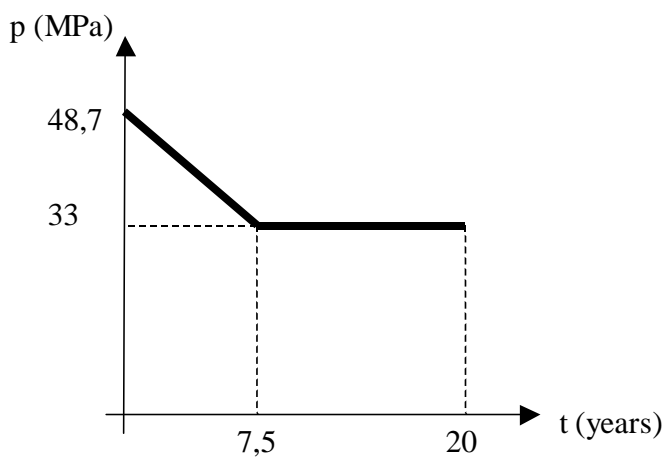

Fig. 7. Exploitation scenario.

\subsection{Results}

Results regarding fluid pressure and fluid flow variation along the fracture are presented in the Fig. 7 and 8 . The curves are related to the pressure after 7.5 and 20 years of simulation.

The important hydraulic role of the fracture is underlined through the flow rate evolution results (Fig. 10). The biggest flow rate outgoing from the well boundary is due to the fracture contribution. After 7.5 years of depletion a fracture flow rate maximum is reached while after, keeping pressure constant for the following 12.5 years of simulation, a small decrease is observed.

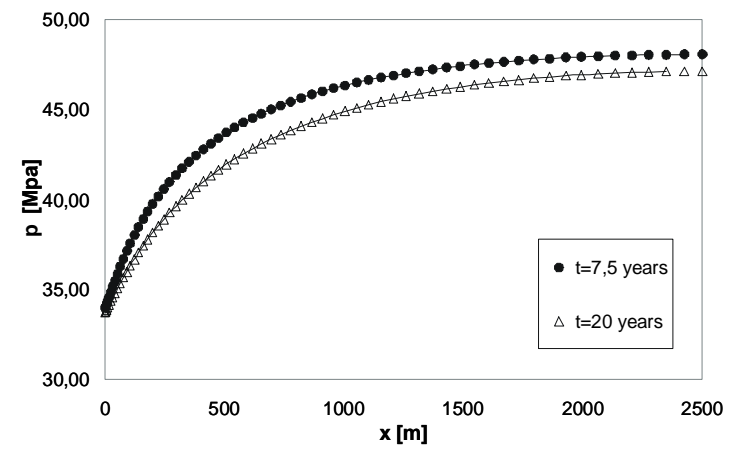

Fig. 8. Fluid pressure along the fracture after 7.5 and 20 years of simulation.

It is also observed (Fig. 10) that the contributions related to the average of the flow rate outgoing from the two rock matrix to the well are coincident and negligible compared to the flow rate associated to the fracture.

Fracture coupled behavior is put in evidence by the following results. Due to the observed fracture fluid pressure decrease, progressive fracture closure is achieved during the calculation. After 7.5 and 20 years of production simulation, Fig. 11 shows that in the well nearby zone a $50 \%$ reduction of the initial 
fracture opening is achieved, the perturbation fading with the distance from the well is in agree with pressure variation trend.

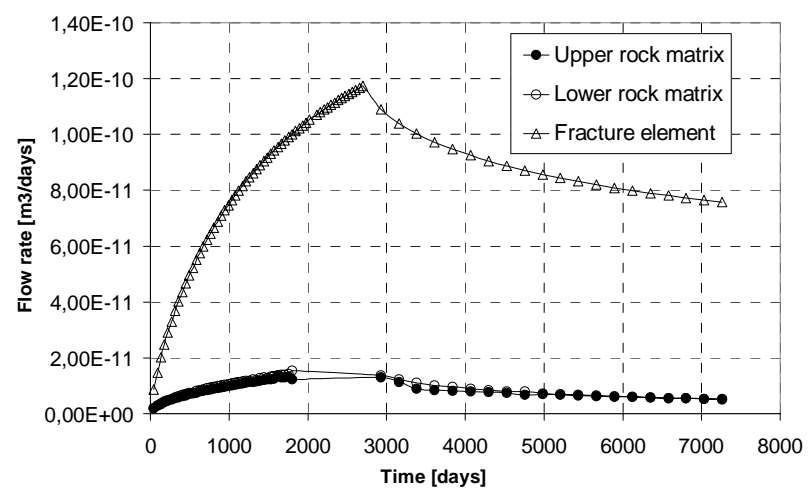

Fig. 9. Outgoing flow rate variation with time increasing.

As shown in Fig. 11, the model succeeds in the representation of fracture hydro-mechanical behavior bounding pressure variation to fracture deformation.

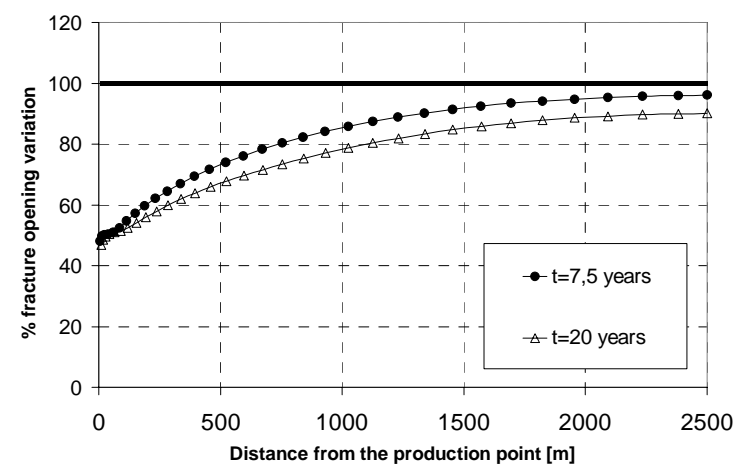

Fig. 10. Fracture opening variation after 7.5 and 20 years of simulation.

The importance of a good fracture description is underlined by comparisons between the presented fracture coupled model and a non-coupled fracture model where opening fracture is maintained constant during all the simulation time.

\subsection{Comparisons}

Two different fracture models were applied for the description of the same reservoir production phase. In particular, results from the precedent computation are compared with those ones obtained from the application of a non-coupled fracture model on the same reservoir schematization using the same initial and boundary conditions. From the comparisons appears that the fracture closure variation, described by the coupled model, heavily influences all the hydraulic parameters.

More in detail, comparisons with the non-coupled model show that the progressive fracture closure is responsible of: 1) a slower fluid pressure decrease along the fracture (Fig. 11) and a lower fluid flow value along all the fracture; 2) a smaller quantity of flow rate outgoing from the fracture to the well boundary (Fig. 12).

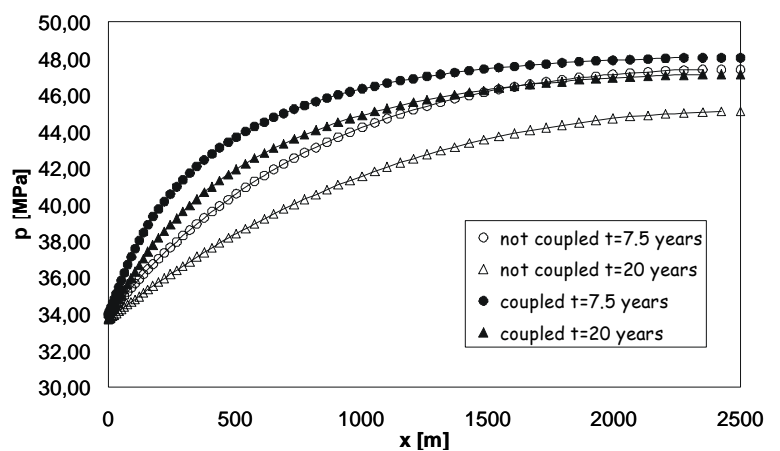

Fig. 11. Fluid pressure - comparison between coupled and non-coupled fracture model.

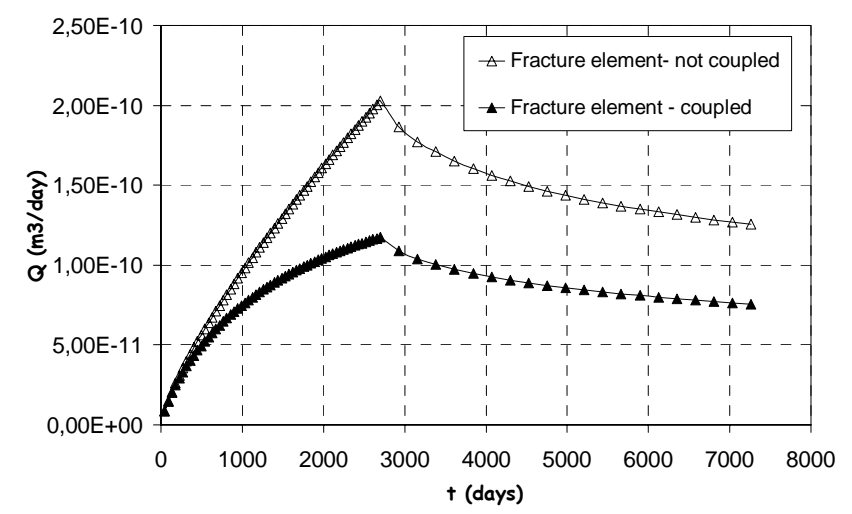

Fig. 12. Flow rate trend comparison using a coupled and a noncoupled fracture model.

\subsection{Sensitivity study}

Two different sensitivity studies were performed in the following to test the correct description given by the presented coupled model. Both studies were performed starting from the same reservoir configuration of the previous simulations. Keeping the same initial conditions, different essays were developed applying different boundary hydraulic conditions. In the first study (Fig. 13) a fluid pressure depletion of $10 \mathrm{MPa}$ was applied at different time intervals (6 months, 1, 7.5 and 15 years). In the second study three essays are developed using three different fluid pressure depletions respectively of 5, 10 and $20 \mathrm{MPa}$ applied at the same time step of 7.5 years (Fig. 13). During both studies, after the respectively depletion phase, pressure is maintained constant until 20 years of simulation time. a)

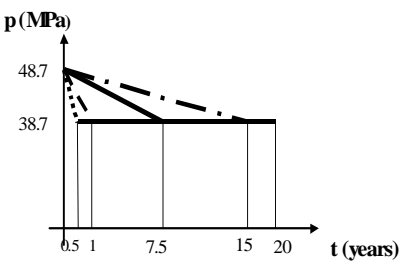

b)



Fig. 13. Hydraulic boundary condition for the first sensitivity case (a) and the second one (b).

Results about the first study show that the highest flow rate peak value is achieved for short time step (6 months) of $10 \mathrm{MPa}$ depletion, while the lowest value is obtained for a $\Delta \mathrm{p}=10 \mathrm{MPa}$ applied during a 
time step of 15 years. So, to faster applications of the fluid pressure variation at the well boundary, it's observed, in the flow rate curves, the presence of higher peaks reached in shorter times (Fig. 14). It's also observed that, at long terms, the different flow rate curves trends related to this study reach more or less the same value.

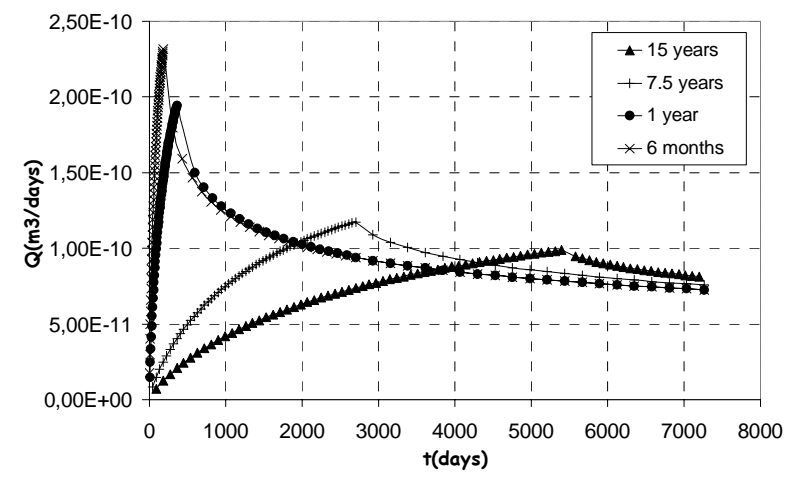

Fig. 14. First sensitivity study results - Different flow rate path applying a $10 \mathrm{MPa}$ depletion respectively in 6 months, 1, 7.5 and 15 years.

Further results on fracture opening illustrate, once more, the direct proportionality between the $\Delta \mathrm{p}$ application velocity and the closure fracture variation.

Second sensitivity study results show, this time, the existence of a non-proportional correspondence between both $\Delta \mathrm{p}$ applied steps with flow rate curves (Fig. 15) and $\Delta \mathrm{p}$ with fracture closure variation.

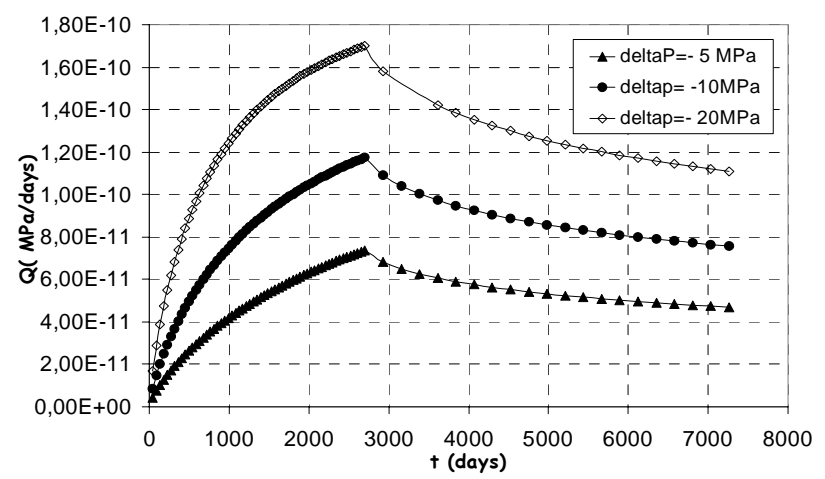

Fig. 15. Second sensitivity study results - Different flow rate path for 5, 10 and $20 \mathrm{MPa}$ imposed pressure variation.

\section{CONCLUSIONS}

A coupled fracture model was developed in this paper to predict the influence of the hydro-mechanical fracture behavior in the oil reservoir depletion. It combines the cubic law with a non-linear deformation fiunction (hyperbolic) suggested to describe the stress-closure/opening curves of the joints.

The model was implemented in the finite element code LAGAMINE in order to be validated.

Academic simulations and comparisons using a non-coupled model were performed to show the two main advantages of the presented methodology. First one, the innovative description of the fracture behav- ior obtained by taking into account both the hydraulic and mechanical aspect. This is in contrast with the actual tendency to consider fractures influence on the reservoir only from an hydraulic point of view. Second one, the attempt to offer a representative description of fractured oil reservoirs through a finite element schematization where only the main fractures were reproduced. These tools avoid the difficulties for a numerical code to reproduce a complex and non-homogeneous fracture field.

Further applications to real fractured oil reservoir geometry will be developed in the future.

\section{REFERENCES}

Bandis, S. Lumsden, A.C. \& Barton, N. 1981. Experimental studies of scale effects on the shear behavior of rock joints. Int. Jour. Of Rock Mechanics and mining Science \& Geomech. Abstr. Vol. 18(1), pp. 1-21.

Bandis, S. Lumsden, A.C. \& Barton, N. 1983. Foundamentals of rock joints deformation. Int. Jour. Of Rock Mechanics and mining Science \& Geomech. Abstr. Vol. 20(6), pp. 249-68.

Bart, M. 2000. Contribution à la modélisation du comportement hydromécanique des massifs rocheux avec fracture. Ph. D. Thesis, Univ. des Sciences et Technologies de Lille.

Barton, N. Choubey, V. 1976. The shear strenght of rock joints in theory and practice. Rock Mechanics and Rock Engineering. Vol. 10, pp. 1-54.

Barton, N. Bandis, S. \& Bakhtar, K. 1985. Strenght deformation and conductivity coupling of rock joints. Int. Jour. Of Rock Mechanics and mining Science \& Geomech. Abstr. Vol. 22(3), pp. 121-140.

Charlier, R. Cescotto, S. 1988. Modélisation du phénomène de contact unilatéral avec frottement dans un contexte de grandes déformations. Jl. Of Theorical and Applied Mechanics (special issue, supplement), Vol. 7(1).

Cescotto, S. Charlier, R. 1993. Frictional contact finite elements based on mixed variational principles. Int. Jl. For Numerical Methods in Engineering, Vol. 36, pp. 16811701.

Goodman, R. Taylor, R. \& Brekke, T. 1968. A model for the mechanics of jointed rock. J. Soil Mech. Fdns Div., Proc. Am. Soc.civ. Engr, № 94, pp. 637-659.

Goodman, R. Debois, J. 1972. Duplication of dilatancy in analysis of jointed rocks. Journal of the soil mechanics and foundations division, ASCE, Vol. 98, SM 4 pp. 399-422.

Goodman, R. 1974. The mechanical properties of joints. Proc. 3D Congr. ISRM, Denver, Vol. 1a, pp. 127-140.

Habraken, A.M. Cescotto, S. 1996. Contact between deformable solids. The fully coupled approach. Jl. For Mathematical and Computer Modelling (special issue: Recent advances in contact and impact mechanics).

Plesha, M. E. 1995. Rock joints: Theory, constitutive equations. Studies in applied mechanics 42, Mechanics of geomaterials interfaces, ELSEVIER, pp. 375-394.

Tsang, Y. W. Witherspoon, P. A. 1981. Hydromechanical behavior of a deformable rock fracture subject to normal stress. J. Geophys. Res. 86, pp. 9287-9298. 